\title{
Hidden conformal symmetry of rotating charged black holes
}

\author{
Deyou Chen 1 , Peng Wang 2, Houwen Wu $3^{3}$ and Haitang Yang 4 \\ School of Physical Electronics, University of Electronic Science and \\ Technology of China, Chengdu, Sichuan 610054, China
}

\begin{abstract}
Motivated by the recent work of the hidden conformal symmetry of the Kerr black hole, we investigate the hidden conformal symmetry of a Kerr-Sen black hole and a Kerr-Newman-Kasuya black hole. Our result shows the conformal symmetry is spontaneously broken due to the periodicity of the azimuthal angle. The absorption across section is in consistence with the finite temperature absorption cross section for a 2D CFT. The entropies of the black holes are reproduced by the Cardy formula.
\end{abstract}

\section{Introduction}

The Kerr/CFT correspondence put forward by Guica, Hartman, Song and Strominger [1] has attracted people's great interest. In this work, quantum gravity in the region very near the horizon of an extreme Kerr black hole was researched. After the specification of boundary conditions at the asymptotic infinity of the near-horizon extreme Kerr (NHEK) geometry and the demonstration of their consistency, the authors showed the asymptotic symmetry group is one copy of the conformal group and furthermore has a central charge $c_{L}=12 \mathrm{~J}$. The macroscopic Bekenstein-Hawking entropy was reproduced by the microscopical computation of entropy from the Cardy formula. Subsequently, this work was extended to various background spacetime and all of the work supports the Kerr/CFT correspondence and give the evidences of holographic duality. The related work can be seen in 3 . In fact, the holographic duality was early studied in the work of Brown and Henneaux [2].

Very recently, Castro and his collaborators [4] have studied the hidden conformal symmetry of the non-extreme Kerr background spacetime by the massless scalar field and showed there is the conformal symmetry, but it is spontaneously broken due to the existence of $2 \pi$ periodicity of the azimuthal

\footnotetext{
${ }^{1}$ E-mail: deyouchen@uestc.edu.cn

${ }^{2}$ E-mail: pengw@uestc.edu.cn

${ }^{3}$ E-mail: iverwu@uestc.edu.cn

${ }^{4}$ E-mail: hyanga@uestc.edu.cn
} 
angle. It should point out that the conformal symmetry is not symmetry of the spacetime geometry and it acts locally on the solution space. The calculation of the absorption probability shows that the near region of the Kerr background spacetime is dual to the 2D CFT. In the microcosmic depiction, using the Cardy formula for the microstate degeneracy, they reobtained the Bekenstein-Hawking entropy of the black hole. Based on this work, Krishnan explored the hidden conformal symmetry of the most general black holes in five dimensions arising from heterotic/type II string theory [5]. Chen and Sun studied the hidden conformal symmetry of the nonextremal uplifted 5D Reissner-Nordstrom black hole [6]. The case of Kerr-Newman black holes was investigated by Wang and Chen in [7] and 8], respectively. Li et.al. investigated the case of the Kaluza-Klein Black Hole [9].

Our aim in this paper is to explore the hidden conformal symmetry of a Kerr-Sen black hole and a Kerr-Newman-Kasuya black hole by using the idea of Castro and his collaborators. Our result is similar to that of the Kerr black hole and the conformal symmetry is spontaneously broken. The Bekenstein-Hawking entropies of the black holes are recovered by the Cardy formula and the CFT temperatures are obtained respectively. Finally, we investigate the absorption across section of the Kerr-Sen black hole in the near region and get the result that the near region of the Kerr-Sen black hole is dual to 2D CFT. The similar case of the Kerr-Newman-Kasuya black hole is investigated.

The rest of this paper is organized as follows. In sect. 2, the hidden conformal symmetry of the Kerr-Sen black hole is explored by the massless scalar field. In sect. 3, we explore the hidden conformal symmetry of the Kerr-Newman-Kasuya black hole. Meanwhile, the entropy and the absorption cross section are investigated. Sect. 4 includes our conclusions.

\section{Hidden Conformal Symmetry of the Kerr-Sen Black Hole}

The Kerr-Sen solution was derived from the low energy effective action for heterotic string theory [11]. In the generalized Boyer-Linquist coordinates, the metric is given by

$d s^{2}=-\frac{\Delta}{\Sigma}\left(d t-a \sin ^{2} \theta d \phi\right)^{2}+\frac{\Sigma}{\Delta} d r^{2}+\Sigma d \theta^{2}+\frac{\sin ^{2} \theta}{\Sigma}\left[a d t-\left(\Sigma+a^{2} \sin ^{2} \theta\right) d \phi\right]^{2}$,

with the electromagnetic potential 


$$
A_{\mu}=\frac{Q r}{\sqrt{2} \Sigma}\left(d t-a \sin ^{2} \theta d \phi\right)
$$

where

$$
\begin{gathered}
\Delta=r^{2}-2 M^{\prime} r+a^{2}=\left(r-r_{+}\right)\left(r-r_{-}\right), \\
\Sigma=r^{2}+2 b r+a^{2} \cos ^{2} \theta, r_{ \pm}=M^{\prime} \pm \sqrt{M^{\prime 2}-a^{2}},
\end{gathered}
$$

$r_{ \pm}$are the outer (inner) horizons and $M^{\prime}=M-b=M-\frac{Q^{2}}{2 M} . M, Q$ and $J=M a$ are physical mass, charge and angular momentum of the black hole, respectively. The horizon area, entropy, Hawking temperature and angular velocity at the event horizon are

$$
\begin{gathered}
A=4 \pi\left(r_{+}^{2}+2 b r_{+}+a^{2}\right)=8 \pi M r_{+}, \\
S=2 \pi M r_{+}, \quad T=\frac{r_{+}-M^{\prime}}{4 \pi M r_{+}}, \quad \Omega_{+}=\frac{a}{2 M r_{+}} .
\end{gathered}
$$

In this paper, we only consider the massless scalar particles. The solution of the Klein-Gordon equation for the massless scalar field on the Kerr-Sen background spacetime is obtained as follows

$$
\begin{gathered}
\partial_{r}\left(\Delta \partial_{r} \Phi\right)-\frac{1}{\Delta}\left[\left(r^{2}+2 b r+a^{2}\right) \partial_{t}+a \partial_{\phi}\right]^{2} \Phi+\frac{1}{\sin \theta} \partial_{\theta}\left(\sin \theta \partial_{\theta} \Phi\right) \\
+\left(a \sin \theta \partial_{t}+\frac{1}{\sin \theta} \partial_{\phi}\right)^{2} \Phi=0 .
\end{gathered}
$$

The existence of two killing vectors $\partial_{t}$ and $\partial_{\phi}$ enables us to separate the variables as $\Phi=e^{-i(\omega t-m \phi)} R(r) \Theta(\theta)$, where $\omega$ and $m$ are quantum numbers. Then the wave equation can be decomposed into two parts (angular part and radial part), which are

$$
\begin{aligned}
-\Lambda \Theta=\frac{1}{\sin \theta} \partial_{\theta}\left(\sin \theta \partial_{\theta} \Theta\right)-\left(\omega^{2} a \sin ^{2} \theta+\frac{m^{2}}{\sin ^{2} \theta} \partial_{\phi}\right) \Theta \\
\Lambda R=\partial_{r}\left(\Delta \partial_{r} R\right)+\frac{\left(m a-2 M r_{+} \omega\right)^{2}}{\left(r-r_{+}\right)\left(r_{+}-r_{-}\right)} R-\frac{\left(m a-2 M r_{-} \omega\right)^{2}}{\left(r-r_{-}\right)\left(r_{+}-r_{-}\right)} R \\
+[\Delta+4 M(M+r)] \omega^{2} R .
\end{aligned}
$$


We are interested in the case of the low frequency limit $\omega M \ll 1$. In this case the geometry can be divided into two parts and the terms included $\omega^{2}$ can be neglected in eqs. (6) and (7). In the near region defined as $r \omega \ll 1$ [4], eqs. (6) and (7) are simplified as

$$
\begin{gathered}
-\Lambda \Theta=\frac{1}{\sin \theta} \partial_{\theta}\left(\sin \theta \partial_{\theta} \Theta\right)-\frac{m^{2}}{\sin ^{2} \theta} \partial_{\phi} \Theta \\
\Lambda R=\partial_{r}\left(\Delta \partial_{r} R\right)+\frac{\left(m a-2 M r_{+} \omega\right)^{2}}{\left(r-r_{+}\right)\left(r_{+}-r_{-}\right)} R-\frac{\left(m a-2 M r_{-} \omega\right)^{2}}{\left(r-r_{-}\right)\left(r_{+}-r_{-}\right)} R .
\end{gathered}
$$

It is obvious to find that eq. (8) denotes the standard Laplacian on the 2sphere. Thus one can get $\Lambda=l(l+1)$. Comparing eq. (9) with the Kerr case, we find they have the similar form, the difference is the values of $r_{ \pm}$.

In fact, Eq. (9) can also be derived from the $S L(2, R)$ Casimir operator, which reflects the $S L(2, R)_{L} \times S L(2, R)_{R}$ symmetry of the near-region scalar field equation. This is improved in the following. We follow Ref. [4, 5, 6, 7, , 8, 9] and firstly introduce the conformal coordinates $\left(\omega^{ \pm}, y\right)$ that relate to the coordinates $(t, r, \phi)$ by

$$
\begin{gathered}
\omega^{+}=\sqrt{\frac{r-r_{+}}{r-r_{-}}} e^{2 \pi T_{R} \phi+2 n_{R} t}, \\
\omega^{-}=\sqrt{\frac{r-r_{+}}{r-r_{-}}} e^{2 \pi T_{L} \phi+2 n_{L} t}, \\
y=\sqrt{\frac{r_{+}-r_{-}}{r-r_{-}}} e^{\pi\left(T_{R}+T_{L}\right) \phi+\left(n_{R}+n_{L}\right) t} .
\end{gathered}
$$

The definition of the local vector field is

$$
\begin{aligned}
& H_{1}=i \partial_{+}, \quad H_{0}=i\left(\omega^{+} \partial_{+}+\frac{1}{2} y \partial_{y}\right), \quad H_{-1}=i\left[\left(\omega^{+}\right)^{2} \partial_{+}+\omega^{+} y \partial_{y}-y^{2} \partial_{-}\right] \\
& \tilde{H}_{1}=i \partial_{-}, \quad \tilde{H}_{0}=i\left(\omega^{-} \partial_{-}+\frac{1}{2} y \partial_{y}\right), \quad \tilde{H}_{-1}=i\left[\left(\omega^{-}\right)^{2} \partial_{-}+\omega^{-} y \partial_{y}-y^{2} \partial_{+}\right]
\end{aligned}
$$


which obey the $S L(2, R)$ Lie bracket algebra

$$
\left[H_{0}, H_{ \pm 1}\right]=\mp i H_{ \pm 1}, \quad\left[H_{-1}, H_{1}\right]=-2 i H_{0},
$$

and the same commutators are hold for $\left(\tilde{H}_{0}, \tilde{H}_{ \pm 1}\right)$. The corresponding quadratic Casimir is

$$
\mathrm{H}^{2}=\tilde{\mathrm{H}}^{2}=-H_{0}^{2}+\frac{1}{2}\left(H_{1} H_{-1}+H_{-1} H_{1}\right)=\frac{1}{4}\left(y^{2} \partial_{y}^{2}-y \partial_{y}\right)+y^{2} \partial_{+} \partial_{-} .
$$

To study the Casimir, we choose the identifications,

$$
\begin{gathered}
n_{L}=-\frac{1}{4 M}, \quad T_{L}=\frac{r_{+}+r_{-}}{4 \pi a}=\frac{2 M^{2}-Q^{2}}{4 \pi M a}, \\
n_{R}=0, \quad T_{R}=\frac{r_{+}-r_{-}}{4 \pi a}=\frac{\sqrt{\left(2 M^{2}-Q^{2}\right)^{2}-4 M^{2} a^{2}}}{4 \pi M a} .
\end{gathered}
$$

From eqs. (10), (14) and (15), the Casimir in term of $(t, r, \phi)$ becomes

$$
\mathrm{H}^{2}=\partial_{r}\left(\Delta \partial_{r}\right)-\frac{\left(a \partial_{\phi}+2 M r_{+} \partial_{t}\right)^{2}}{\left(r-r_{+}\right)\left(r_{+}-r_{-}\right)}+\frac{\left(a \partial_{\phi}+2 M r_{-} \partial_{t}\right)^{2}}{\left(r-r_{-}\right)\left(r_{+}-r_{-}\right)} .
$$

Comparing eq. (9) with eq. (16), we find the wave of radial part in the near region can be rewritten as

$$
\mathrm{H}^{2} \Phi=\tilde{\mathrm{H}}^{2} \Phi=l(l+1) \Phi,
$$

which shows the scalar Laplacian can be reduced to the $S L(2, R)$ Casimir and the $S L(2, R)_{L} \times S L(2, R)_{R}$ weights of the field $\Phi$ are $(l, l)$. We know the vector fields defined in eqs. (11) and (12) are local and the angular $\phi$ is periodic. Therefore they are not periodic under the angular identification $\phi \sim \phi+2 \pi$. This implies that the $S L(2, R)_{L} \times S L(2, R)_{R}$ symmetry is spontaneously broken under the periodic identification of the angular coordinate $\phi$.

In the following, we reproduce the Bekenstein-Hawking entropy of the Kerr-Sen black hole from the Cardy formula for the dual 2D CFT. There are many papers about the black hole entropy. The attractor mechanism plays an important role when the scalars are turned on (black holes in string theory). The research on the attractor mechanism and entropy of extremal black holes based on the entropy function formalism has been discussed [10]. 
Here, we don't know the corresponding the central charge $C_{L}$ and $C_{R}$ of the non-extreme Kerr-Sen black hole. In Ref. [4, 5, 6, 7, 8, 9] the central charges near the extreme case are adopted and are regarded as that of the nonextreme case. The reason is that there is a smooth limit from near-extremal to extremal solution and probably the hidden conformal symmetry connects smoothly to that of extreme limit 5 . Therefore we can get the central charges from the case of the extreme Kerr-Sen black hole, which is

$$
C_{L}=C_{R}=12 J
$$

From the Cardy formula for the microstate degeneracy, we get

$$
S=\frac{\pi^{2}}{3}\left(C_{L} T_{L}+C_{R} T_{R}\right)=2 \pi M r_{+}=S_{B H},
$$

where eq. (15) is introduced to get the second equal sign and which shows the Bekenstein-Hawking entropy can be derived from the dual 2D CFT.

Now we solve Eq. (7). There is much work appeared to solve such equation in the research on greybody factors of black holes at the frequency $\omega M \ll 1$ [[12] and the references therein]. The difference here is that our consideration is the solution in the near region $\omega r \ll 1$. To bring Eq. (7) in the form of a known differential equation, we introduce the following transformation [8, 12 ]

$$
f=\frac{r-r_{-}}{r-r_{+}}
$$

And then eq.(7) becomes

$f(1-f) \frac{d^{2} R}{d f^{2}}+(1-f) \frac{d R}{d f}+\left\{\frac{\left[m a-2 M r_{+} \omega\right]^{2}}{f\left(r_{+}-r_{-}\right)^{2}}-\frac{\left[m a-2 M r_{-} \omega\right]^{2}}{\left(r_{+}-r_{-}\right)^{2}}-\frac{l(l+1)}{1-f}\right\} R=0$

It is not a simply work to solve the above equation. We can follow the method given in [12] and get the solution. So the absorption cross section is

$$
P_{a c s} \sim|A|^{-2}=\sinh \left(\frac{\omega-m \Omega}{2 T}\right)|\Gamma(l+1-i 2 M \omega)|^{2}
$$

\footnotetext{
${ }^{5}$ We are grateful to the reviewer for his help on this point.
} 


$$
\cdot\left|\Gamma\left(l+1-i 2 \frac{M \omega\left(r_{+}+r_{-}\right)-m a}{r_{+}-r_{-}}\right)\right|^{2} .
$$

We find it has similar form with that of the Kerr-Newman case, but the values of $r_{ \pm}$are different. We show that this absorption across section relates to a 2D CFT in the following. To do this, we should first find the conjugate charges $\left(E_{L}, E_{R}\right)$ corresponding to the CFT temperatures given by eq. (15), which satisfy the relation

$$
\delta S=\frac{\delta E_{L}}{T_{L}}+\frac{\delta E_{R}}{T_{R}} .
$$

The solution of the conjugate charges is easily gotten as

$$
\delta E_{L}=\frac{\delta M}{a}\left(r_{+}+r_{-}\right) M, \quad \delta E_{R}=\frac{\delta M}{a}\left(r_{+}+r_{-}\right) M-\delta J
$$

after the first law of thermodynamics $T_{H} \delta S=\delta M-\Omega_{H} \delta J-\Phi_{H} Q$ was introduced. In [8], the conjugate charges was derived with the consideration of electro-magnetic field, where $\Phi_{H} Q \neq 0$. Since the massless scalar field is considered in this paper, we do not care $\Phi_{H} Q$. Hence the left and right moving frequencies are

$$
\omega_{L}=\frac{\omega}{a}\left(r_{+}+r_{-}\right) M, \quad \omega_{R}=\frac{\omega}{a}\left(r_{+}+r_{-}\right) M-m .
$$

where $\delta M=\omega$ and $\delta J=m$ were used here. Combining eqs.(15), (22), (25) and the weights $(l, l)$, we can get the absorption cross section as follows

$P \sim T_{L}^{2 h_{L}-1} T_{R}^{2 h_{R}-1} \sinh \left(\frac{\omega_{L}}{T_{L}}+\frac{\omega_{R}}{T_{R}}\right)\left|\Gamma\left(h_{L}+i \frac{\omega_{L}}{2 \pi T_{L}}\right)\right|^{2} \cdot\left|\Gamma\left(h_{R}+i \frac{\omega_{R}}{2 \pi T_{R}}\right)\right|^{2}$

which is just the finite-temperature absorption cross section for a $2 \mathrm{D}$ CFT and shows the near region of the Kerr-Sen background spacetime is dual to 2D CFT.

\section{Hidden Conformal Symmetry of a Kerr-Newman-Kasuya} black hole

The hidden conformal symmetry of the charged rotating black hole has been investigated [7, 8]. In this section, we investigate the hidden conformal symmetry of a rotating black hole with electric charge and magnetic charge 
(namely a Kerr-Newman-Kasuya black hole) [13]. The metric in the BoyerLindquist coordinates is given by

$$
\begin{gathered}
d s^{2}=-\left(1-\frac{2 M r-Q^{2}-P^{2}}{\sum}\right) d t^{2}-\frac{2 a \sin ^{2} \theta}{\sum}\left(2 M r-Q^{2}-P^{2}\right) d t d \varphi \\
+\frac{\sum}{\Delta} d r^{2}+\sum d \theta^{2}+\left(\frac{\left(2 M r-Q^{2}-P^{2}\right) a^{2} \sin ^{2} \theta}{\sum}-\left(r^{2}+a^{2}\right)\right) \sin ^{2} \theta d \varphi^{2},
\end{gathered}
$$

with the electro-magnetic vector potential

$$
A=\frac{Q r}{\sum}\left(d t-a \sin ^{2} \theta d \phi\right)+\frac{P \cos \theta}{\sum}\left[a d t-\left(r^{2}+a^{2}\right) d \phi\right]+\varepsilon P d \phi,
$$

where

$$
\begin{gathered}
\Delta=r^{2}-2 M r+a^{2}+Q^{2}+P^{2}=\left(r-r_{+}\right)\left(r-r_{-}\right), \\
\sum=r^{2}+a^{2} \cos ^{2} \theta, \quad \varepsilon= \pm 1,0, \quad r_{ \pm}=M \pm \sqrt{M^{2}-a^{2}-Q^{2}-P^{2}} .
\end{gathered}
$$

parameters $M, Q$ and $P$ are the physical mass, electric charge and magnetic charge of the black hole, respectively. The horizon area, entropy, Hawking temperature and angular velocity at the event horizon are

$$
\begin{gathered}
A=4 \pi\left(2 M r_{+}-Q^{2}-P^{2}\right), \quad S=\pi\left(2 M r_{+}-Q^{2}-P^{2}\right), \\
T_{H}=\frac{r_{+}-r_{-}}{4 \pi\left(r_{+}^{2}+a^{2}\right)}, \quad \Omega_{H}=\frac{a}{\left(r_{+}^{2}+a^{2}\right)} .
\end{gathered}
$$

Since the Kerr-Newman-Kasuya metric can be obtained from Kerr-Newman one by replacing $Q^{2}$ by $Q^{2}+P^{2}$, all the calculations are parallel to those of Kerr-Newman, investigated by [8], we simply list the final conclusion here.

- Once considering a massless scalar field, the Laplacian can be reduced to the $S L(2, R)$ Casimir in the near region $r \omega \ll 1$. 
- With Cardy's formula and the available constraints $C_{L}=C_{R}=12 \mathrm{~J}$, the CFT microstate degeneracy precisely coincides the BekensteinHawking entropy of the Kerr-Newman-Kasuya:

$$
S=\frac{\pi^{2}}{3}\left(C_{L} T_{L}+C_{R} T_{R}\right)=\pi\left(2 M r_{+}-Q^{2}-P^{2}\right),
$$

- In the near region, after imposing $r \gg M$, similar calculation as in [8] shows that

$$
\begin{gathered}
p_{a b s}=|A|^{-2} \sim \sinh \frac{\omega-m \Omega_{H}}{2 T_{H}}|\Gamma(l+1-i 2 M \omega)|^{2} \\
.\left|\Gamma\left(l+1+i 2 \frac{m a-\left(2 M^{2}-Q^{2}-P^{2}\right) \omega}{r_{+}-r_{-}}\right)\right|^{2} .
\end{gathered}
$$

With identifications of the corresponding left and right moving frequencies

$$
\begin{gathered}
\omega_{L}=\frac{2 M^{2}-Q^{2}-P^{2}}{a} \omega, \\
\omega_{R}=\frac{2 M^{2}-Q^{2}-P^{2}}{a} \omega-m,
\end{gathered}
$$

the two dimensional CFT finite-temperature absorption cross section is reproduced:

$$
p_{a b s} \sim T_{L}^{2 h_{L}-1} T_{R}^{2 h_{R}-1} \sinh \left(\frac{\omega_{L}}{2 T_{L}}+\frac{\omega_{R}}{2 T_{R}}\right)\left|\Gamma\left(h_{L}+i \frac{\omega_{L}}{2 \pi T_{L}}\right)\right|^{2} \cdot\left|\Gamma\left(h_{R}+i \frac{\omega_{R}}{2 \pi T_{R}}\right)\right|^{2},
$$

\section{Conclusions}

In this paper, we have investigated the the dual conformal field theory of the Kerr-Sen black hole and the Kerr-Newman-Kasuya black hole. The result shows there is spontaneous breaking of the conformal symmetry due to the existence of the periodic identification of the azimuthal angle $\phi$ in the background spacetime of the black holes. For the Kerr-Sen black hole, we get the CFT temperatures as $T_{L}=\frac{2 M^{2}-Q^{2}}{4 \pi M a}$ and $T_{R}=\frac{\sqrt{\left(2 M^{2}-Q^{2}\right)^{2}-4 M^{2} a^{2}}}{4 \pi M a}$. For the Kerr-Newman-Kasuya black hole, the CFT temperatures are $T_{L}=$ 
$\frac{2 M^{2}-Q^{2}-P^{2}}{4 \pi M a}$ and $T_{R}=\frac{\sqrt{M^{2}-Q^{2}-P^{2}-a^{2}}}{2 \pi a}$. Meanwhile the Bekenstein-Hawking entropies of the black holes were reobtained by the Cardy formula. When the conjugate charges and the CFT temperatures were introduced, we find the absorption cross section of the Kerr-Sen black hole is just the finite temperature absorption cross section for a 2D CFT. There is the same case for the Kerr-Newman-Kasuya black hole. This shows the absorption across section in gravity is in consistency with that in CFT and the near region of the background spacetime of the black hole is dual to 2D CFT.

\section{Acknowledgments}

One of the authors (D. Chen) would like to thank Alejandra Castro and Chiang-Mei Chen for their help. This work is supported by Fundamental Research Funds for the Central Universities (Grant No. ZYGX2009J044, ZYGX2009X008), NSFC (Grant No.10705008) and NCET.

\section{References}

[1] M. Guica, T. Hartman, W. Song and A. Strominger, "The Kerr/CFT Correspondence", arXiv: 0809.4266[hep-th].

[2] J.D. Brown and M. Henneaux, "Central Charges in the Canonical Realization of Asymptotic Symmetries: An Example from ThreeDimensional Gravity", Commun. Math. Phys. 104 (1986) 207.

[3] K. Hotta, Y. Hyakutake, T. Kubota, T. Nishinaka and H. Tanida, "The CFT-interpolating black hole in three dimensions", JHEP 0901 (2009) 010, arXiv:0811.0910 [hep-th]];

H. Lu, J. Mei and C.N. Pope, "Kerr-AdS/CFT Correspondence in Diverse Dimensions", JHEP 0904 (2009) 054, [arXiv:0811.2225 [hep-th]];

T. Azeyanagi, N. Ogawa, and S. Terashima, "Holographic Duals of Kaluza-Klein Black Holes", arXiv:0811.4177 [hep-th]];

T. Hartman, K. Murata, T. Nishioka and A. Strominger, "CFT Duals for Extreme Black Holes", JHEP 0904 (2009) 019, arXiv:0811.4393 [hepth]]; 
Y. Nakayama, "Emerging AdS from Extremally Rotating NS5-branes", Phys. Lett. B 673 (2009) 272, arXiv:0812.2234 [hep-th]];

David D.K. Chow, M. Cvetic, H. Lu and C.N. Pope, Phys. Rev. D 79 (2009) 084018;

H. Isono, T.S. Tai and W.Y. Wen, Int. J. Mod. Phys. A 24 (2009) 5659;

T. Azeyanagi, N. Ogawa and S. Terashima, Phys. Rev. D 79 (2009) 106009;

J.J Peng and S.Q. Wu, Phys. Lett. B 673 (2009) 216; Nucl. Phys. B 828 (2010) 273;

S.M. Carroll, M.C. Johnson and L. Randall, JHEP 0911 (2009) 109;

D. Astefanesei and Y.K. Srivastava, Nucl. Phys. B 822 (2009) 283, arXiv:0902.4033 [hep-th]];

D. Astefanesei and H. Yavartanoo, Nucl. Phys. B 794 (2008) 13, arXiv:0706.1847 [hep-th]];

C. Krishnan and S. Kuperstein, Phys. Lett. B 677 (2009) 326, arXiv:0903.2169[hep-th]];

F. Loran and H. Soltanpanahi, Class. Quant. Grav. 26 (2009) 155019;

A.M. Ghezelbash, JHEP 0908 (2009) 045;

H. Lu, J. Mei, C.N. Pope, J. Vazquez-Poritz, Phys. Lett. B 73 (2009) 77 ;

G. Compere, K. Murata and T. Nishioka, JHEP 0905(2009) 077;

K. Hotta, Phys. Rev. D 79 (2009) 104018;

M.R. Garousi and A. Ghodsi, "The RN/CFT Correspondence", arXiv:0902.4387 [hep-th]];

A.M. Ghezelbash, "Kerr-Bolt Spacetimes and Kerr/CFT Correspondence ", arXiv:0902.4662 [hep-th]];

W.Y. Wen, "Holographic descriptions of (near-)extremal black holes in five dimensional minimal supergravity", [arXiv:0902.4662 [hep-th]];

T. Azeyanagi, G. Compere, N. Ogawa, Y. Tachikawa and S. Terashima, Prog. Theor. hys. 122 (2009) 355;

X.N. W and Y. Tian, Phys. Rev. D 80 (2009) 024014; 
T. Nishioka, S. Ryu and T. Takayanagi, J. Phys. A 42 (2009) 504008;

L.M. Cao, Y. Matsuo, T. Tsukioka and C.M. Yoo, Phys. Lett. B 679 (2009) 390;

O.J.C. Dias, H.S. Reall and J.E. Santos, JHEP 0908 (2009) 101;

A.J. Amsel, G.T. Horowitz, D. Marolf and M.M. Roberts, JHEP 0909 (2009) 044;

Y. Matsuo, T. Tsukioka and C.M. Yoo, Nucl. Phys. B 825 (2010) 231; "Yet Another Realization of Kerr/CFT Correspondence", arXiv:0907.4272 [hep-th]];

I.Bredberg, T. Hartman, W. Song and A. Strominger, "Black Hole Superradiance From Kerr/CFT", arXiv:0907.3477[hep-th]];

A.J. Amsel, D.Marolf and M.M. Roberts, "On the Stress Tensor of Kerr/CFT", arXiv:0907.3477[hep-th]];

B. Chen, Z. Xu, JHEP 0911 (2009) 091;

J. Rasmussen, Int. J. Mod. Phys. A 25 (2010) 1597;

A. Castro and F. Larsen, JHEP 0912 (2009) 037;

M. Cvetic and F. Larsen, JHEP 0909 (2009) 088;

T. Hartman, W. Song and A. Strominger, JHEP 1003 (2010) 118;

V. Jejjala and S. Nampuri, JHEP 1002 (2010) 088;

J. Rasmussen, "A note on Kerr/CFT and free fields", arXiv:0909.2924[hep-th]];

C.M. Chen, J.R. Sun and S.J. Zou, JHEP 1001 (2010) 057;

D. Anninos and T. Hartman, JHEP 1003 (2010) 096;

H. Soltanpanahi, "Near Horizon of 5D Rotating Black Holes from 2D Perspective", arXiv:0911.0148[hep-th]];

B. Chen, B. Ning and Z. Xu, "Real-time correlators in warped AdS/CFT correspondence", arXiv:0911.0167[hep-th]];

B. Chen and C.S. Chu, "RReal-time correlators in Kerr/CFT correspondence", arXiv:1001.3208 [hep-th]];

J.W. Mei, "The Entropy for General Extremal Black Holes", arXiv:1002.1349 [hep-th]]; 
[4] A. Castro, A. Maloney and A. Strominger, "Hidden Conformal Symmetry of the Kerr Black Hole", arXiv: 1004.0996[hep-th].

[5] C. Krishnan, "Hidden Conformal Symmetries of Five-Dimensional Black Holes", arXiv: 1004.3537[hep-th].

[6] C.M. Chen and J.R. Sun, "Hidden Conformal Symmetry of the ReissnerNordstrom Black Holes", arXiv: 1004.3963[hep-th].

[7] Y.Q. Wang andY.X. Liu, "Hidden Conformal Symmetry of the KerrNewman Black Hole", arXiv: 1004.4661[hep-th].

[8] B. Chen and J. Long, "Real-time Correlators and Hidden Conformal Symmetry in Kerr/CFT Correspondence", arXiv: 1004.5039[hep-th].

[9] R. Li, M.F. Li, J.R. Ren, "Entropy of Kaluza-Klein Black Hole from Kerr/CFT Correspondence", arXiv: 1004.5335[hep-th].

[10] A. Sen, Gen. Rel. Grav. 40 (2008) 2249 (arXiv:0708.1270).

[11] A. Sen, Phys. Rev. Lett. 69 (1992) 1006; A. Garcia, D. Galtsov and O. Kechkin, Phys. Rev. Lett. 74 (1995) 1276.

[12] I.R. Klebanov and S.D. Mathur, Nucl. Phys. B 500 (1997) 115;

S.Creek, O. Efthimiou, P. Kanti and K. Tamvakis, Phys. Rev. D 75 (2007) 084043, arXiv:0701288[hep-th];

S. Creek, O. Efthimiou, P.Kanti, K. Tamvakis, Phys. Rev. D 76 (2007) 104013, arXiv:0701288[hep-th].

[13] M. Kasuya, Phys. Rev. D 25 (1982) 995. 